\title{
Electromigration failure by shape change of voids in bamboo lines
}

\author{
E. Arzt and O. Kraft. \\ Max-Planck-Institut für Metallforschung and Universität Stuttgart, D-70174 Stuttgart, Germany \\ W. D. Nix \\ Department of Materials Science and Engineering, Stanford University, Stanford, California 94305 \\ J. E. Sanchez, Jr. \\ Advanced Micro Devices, Sunnyvale, California 94055
}

(Received 16 March '1994; accepted for publication 19 April 1994)

\begin{abstract}
The behavior of electromigration-induced voids in narrow, unpassivated aluminum interconnects is examined, using scanning electron microscopy. Some electromigration tests were interrupted several times in order to observe void nucleation, void growth, and finally the failure of the conductor line. It is found that voids which opened the line have a specific asymmetric shape with respect to the electron flow direction. Besides void nucleation and void growth, void shape changes can consume a major part of the lifetime of the conductor line. A first attempt to model these processes on the basis of diffusion along the void surface shows that voids with a noncircular initial shape tend to produce the fatal asymmetry due to electron wind effects, with the anisotropy of surface energy possibly playing only a minor role.
\end{abstract}

\section{INTRODUCTION}

Failure of metallic interconnects in integrated circuits has long been a key reliability concern, which is further accentuated by the continuing trend toward miniaturization. One possible reason for such failures involves the phenomenon of electromigration, a mechanism for transport of matter by high electric current densities (see, e.g., Ref. 1) which produces damage in the lines. Failure results either from voids growing over the entire line width or extrusions which cause short circuits to neighboring lines.

Modeling the micromechanisms of void formation and growth is a long-standing scientific problem; for a recent review see Ref. 2 . In order to be useful for applications, the final aim must be not only to understand the origins of these processes, but also to predict the time-to-failure distribution. This is still a formidable task although the basics of electron wind effects on diffusion appear to be well understood. Also theoretical analyses exist concerning void nucleation by stress accumulation, e.g., Refs. 3 and 4 or quasi-static void growth, see, e.g., Refs. 5 and 6.

Experimental observations of the damage development, especially recent studies on lines with near-bamboo grain structure, have revealed several complications which need to be considered if the damage processes are to be modeled realistically.

First, voids are not static but rather show motion, usually in the direction opposing the electron wind. This has been confirmed by several in situ electron microscopy studies, employing, e.g., scanning electron microscopy (SEM) on unpassivated Al lines, ${ }^{7,8}$ and field-emission SEM or scanning transmission electron microscopy (STEM), imaging backscattered electrons, on passivated $\mathrm{Al}$ lines. ${ }^{9-11}$ It was also observed that voids can "heal" by breaking up into smaller fragments or grow by coalescing with other voids. ${ }^{11}$

Second, besides "classical" grain boundary diffusion, surface and interface diffusion can contribute to the damage development. This is suggested by in situ transmission elec- tron microscopy (TEM) studies on large grained Al stripes and films revealing voids inside the grains. ${ }^{12}$ These voids had grown in the direction of current flow lines, sometimes without apparently interacting with grain boundaries. Thinning of large regions within a single grain in an Al film during electromigration testing was also observed. ${ }^{13}$ Both observations cannot be explained if grain boundaries are the only diffusion paths.

Third, voids do not grow in a self-similiar manner, but can show significant shape changes. This point has been especially emphasized as a result of our recent electromigration tests which were interrupted several times for damage characterization in an SEM. ${ }^{14}$ A typical void shape has been identified which appears to be necessary for the development of a failure site. The resulting fatal void often has a slit-like morphology, which gives the appearance of a crack perpendicular to the line. Following detailed study by SEM and focused ion beam techniques ${ }^{15,16}$ and by TEM, ${ }^{17,18}$ it seems now well established that these slits frequently do not follow grain boundaries, as might be expected, but are transgranular. Again these observations indicate the necessity to consider mass transport mechanisms other than grain boundary diffusion.

From these experimental studies it can be concluded that electromigration failure is the result of a complicated competition between growth, shape change, and motion of voids. The interaction between these mechanisms is not well understood, although several important attempts have been made to model such events. Void motion has been treated, for example by $\mathrm{Ho},{ }^{19}$ with the result that small voids migrate more rapidly. More recently, Nix and $\mathrm{Arzt}^{20}$ have suggested that a critical void size exists for which void motion is a minimum; the consequence could be that larger voids catch up with smaller ones, moving more rapidly as they do so and resulting in a catastrophic mechanism of void growth and failure. As described by Børgesen et al., ${ }^{21}$ grain boundaries can trap voids until they reach a critical size. Comparatively little has 
been published on alternative diffusion paths besides grain boundary diffusion, and on shape change effects. In any case, none of the published models considers all of the above described mechanisms, but this might be necessary in order to safely extrapolate median time-to-failure (MTF) data, taken under accelerated testing conditions, to operation conditions.

In this article we focus on the shape change effect. Results of a detailed investigation of damage evolution involving more than 500 failure sites on bamboo-structure lines are presented. The observations point to the importance of understanding void shape changes in order to realistically model the failure events and, eventually, to predict the timeto-failure. A first attempt to theoretically explain void shape changes under the action of an electron wind is also presented.

\section{EXPERIMENT}

Accelerated electromigration tests were performed on unpassivated circuits comprising 20 parallel lines. ${ }^{22,23}$ The metallizations consisted of pure aluminum films of $0.5 \mu \mathrm{m}$ thickness, which were sputter deposited and then treated by two different annealing procedures. One was a standard oven anneal at $450{ }^{\circ} \mathrm{C}$ in forming gas ("standard metallization"). The resulting grains had an average size of $1.4 \mu \mathrm{m}$ and a (111) fiber texture with the fraction of (111) grains exceeding $90 \%$. The other annealing procedure was a laser reflow process, ${ }^{24}$ which influenced the microstructure of the film in several ways. Grains were coarsened to approximately $3 \mu \mathrm{m}$ in diameter and grain boundary grooves formed, which have the benefit that the grain boundaries are visible in the SEM withoul any further preparation; the typical (111) fiber texture was destroyed, the "reflowed" film consisted of almost randomly oriented grains [fraction of (111) grains is $\sim 40 \%$ ]. Standard lithographic and etch techniques were used to pattern the samples. The widths of the tested lines were between 1.0 and $5.0 \mu \mathrm{m}$. The electromigration tests were performed with diced chips on the hot chuck of a manual probe station. Constant voltage conditions were applied to prevent destruction of the failure site when an opening in the line occurred. Current densilies were in the range of $1.0-2.2 \mathrm{MA} / \mathrm{cm}^{2}$, the temperature was held constant at $500 \mathrm{~K}$. The temperature increase due to Joule heating was monitored by measuring the resistance change during stepwise increase of the current to the testing condition. For current densities below 2 $\mathrm{MA} / \mathrm{cm}^{2}$ the temperature increase did not exceed $2 \mathrm{~K}$. For current densities in the range $2-2.2 \mathrm{MA} / \mathrm{cm}^{2}$ the increase was between 2 and $5 \mathrm{~K}$ depending on the line width. However, the failure mechanism did not change, and the voids were still randomly distribuled over the enlire line length.

All tested samples were investigated post mortem in an SEM. Some tests were interrupted several times for examination of the sample in order to gain information on void nucleation, motion, and growth. As a measure of void size, the void area was determined on the SEM micrographs, using a bitpad. The area was directly proportional to the void volume because typically the voids extended to the substrate and their sidewalls were perpendicular to the film surface. Complementary to the SEM investigations, some samples were examined using a focused ion beam microscope. This techniques visualizes grain orientations and therefore allows determination of the grain structure. ${ }^{25}$

\section{RESULTS AND DISCUSSION}

Figure 1 shows a typical sequence of void growth in a 1.8- $\mu \mathrm{m}$-wide line of the standard metallization stressed with a current density of $1.6 \mathrm{MA} / \mathrm{cm}^{2}$. These micrographs were taken during interruptions of the test after $24.3,44.5,65.8$, $131.8,177.2$, and $190.1 \mathrm{~h}$ of testing. In all of these and the following micrographs, the direction of the electron flow is from right to left. In Fig. 1(g) the void area is plotted as a function of time. Linear growth is observed with a small incubation period of about $8 \mathrm{~h}$.

Figure 2 shows the development of another typical damage site in a different line during the same test. Here the void is observed for the first time after $65.8 \mathrm{~h}$, then it grows linearly with time along the edge of the line [Figs. 2(a)-2(e) and $2(\mathrm{~h})]$. The big white spot on the line, probably alumina on the surface, can serve as a stationary marker; it is invisible in the SEM if the Al beneath it has been removed by electromigration. In this example, the void breaks into two [Fig. 2(f)], with the total void volume remaining about constant. Although the void on the left of Fig. 2(f) grows to over 80\% of the line width, failure does not occur at this site. Contrary to this, the void on the right moves several microns and grows at the expense of the other void. Figure 2(h) shows the void area as a function of time. Again, void nucleation takes up only a small fraction of the total lifetime and void growth is linear in time: In contrast to Fig. 1, the line does not fail after the void has reached a certain size. Instead, the total void volume, which has remained about constant after $350 \mathrm{~h}$, has to be rearranged to give a shape change which provokes the failure after $780.6 \mathrm{~h}$.

Comparing the two fatal voids in Figs. 1(f) and 2(g), it is striking that the void contour which is turned against the direction of the electron flow is more or less perpendicular to the line. In the course of our investigations more than 500 fatal voids in lines of various widths and alloy composition were investigated and this shape asymmetry was a common observation, especially in narrower lines.

A third sequence is shown in Fig. 3: Here the lines, produced from a laser reflowed film, exhibited a nearbamboo structure; the linewidth amounted to $1.8 \mu \mathrm{m}$ and the applied current density to $2 \mathrm{MA} / \mathrm{cm}^{2}$. Figure 3(a) depicts a void and the corresponding hillock after $12.2 \mathrm{~h}$, both of which had not been observed after $9.4 \mathrm{~h}$. Grain boundary grooves, which are partly visible in the SEM, are highlighted. After $16.5 \mathrm{~h} \mathrm{(b)} \mathrm{and} 25.5 \mathrm{~h}$ (c) the void shape has not changed significantly, only the angle between the cathode (right) boundary of the void and the line changes from $40^{\circ}$ (a) to $10^{\circ}(\mathrm{c})$, indicating that this boundary probably does not reflect a particular crystallographic plane. Figure 3(d) shows the final void shape after $42.2 \mathrm{~h}$, which is totally different from those before. The void grows linearly with time [as shown in Fig. 3(e)], but its shape changes simultaneously. The cathode boundary is neither straight nor perpendicular to the line all the way, but the left part of the void, where the opening occurred, could be approximated as a wedge whose 
(a)
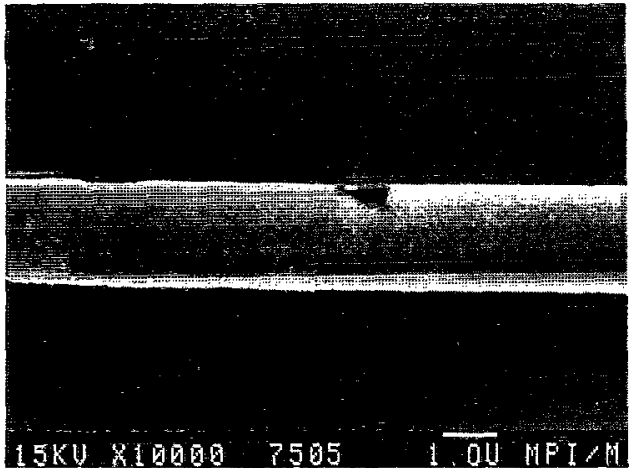

(b)

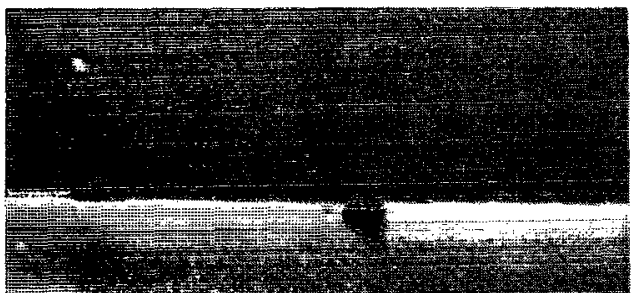

)
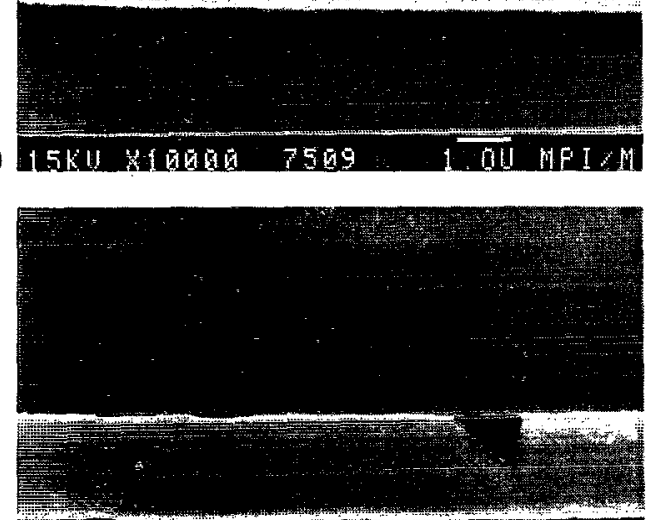

(c)

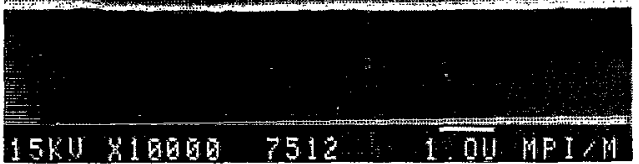

(d)

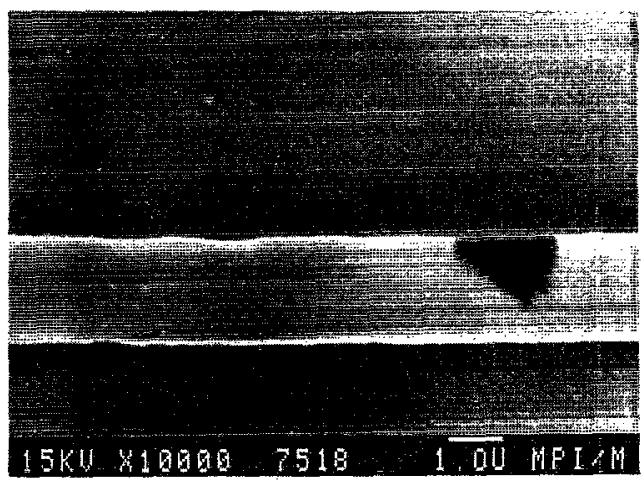

(e)
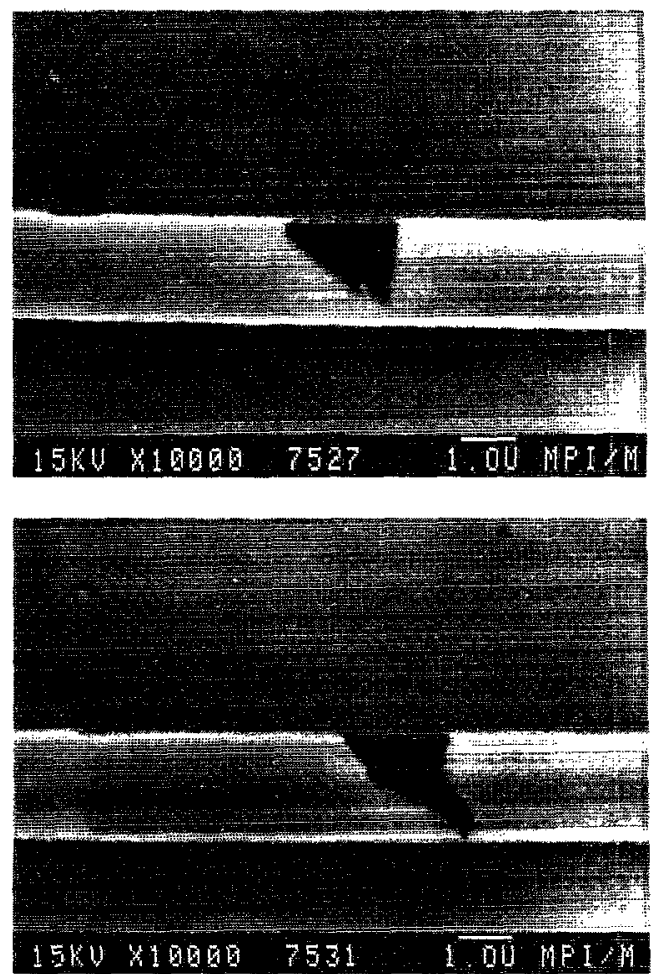

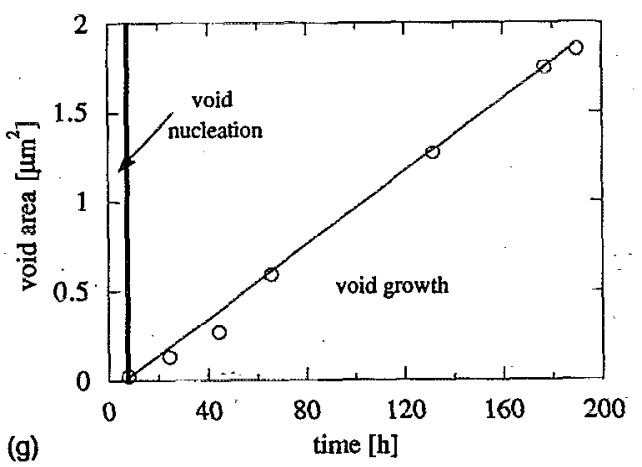

FIG. 1. SEM micrographs showing a time sequence of the same site on a conductor line after (a) $24.3 \mathrm{~h}$, (b) $44.5 \mathrm{~h}$, (c) $65.8 \mathrm{~h}$, (d) $131.8 \mathrm{~h}$, (c) $177.2 \mathrm{~h}$, and (f) $190.1 \mathrm{~h}$. (g) Void area plotted as a function of time.

anode sidewall is oriented at a more acute angle to the line than the cathode one. This sample was examined using the focused ion beam technique in order to visualize the grain structure and it was confirmed that the grain boundaries had not moved under their grooves and the opening had not occurred along grain boundaries.
Our main observation, which we think is significant for the failure mechanism, pertains to the fact that fatal voids have a typical slit or wedge shape with a pronounced asymmetry. ${ }^{14}$ Figure 4 illustrates this observation schematically: Fig. 4(a) shows a void with a straight cathode boundary oriented perpendicular to the line. An opposite boundary 
(a)

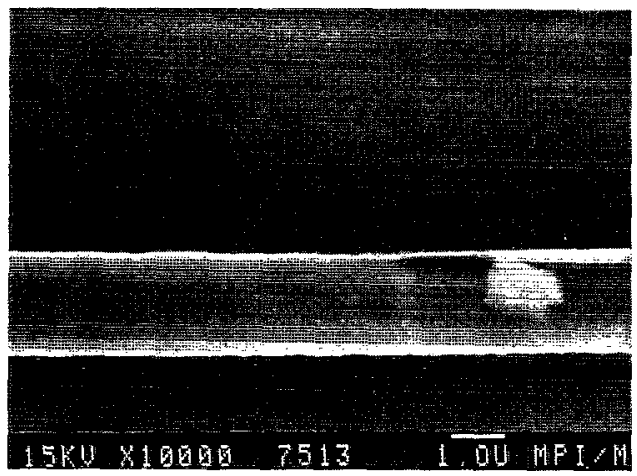

(b)
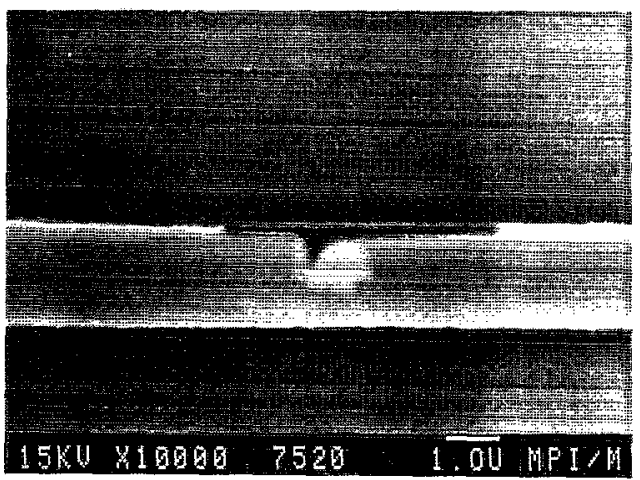

(c)

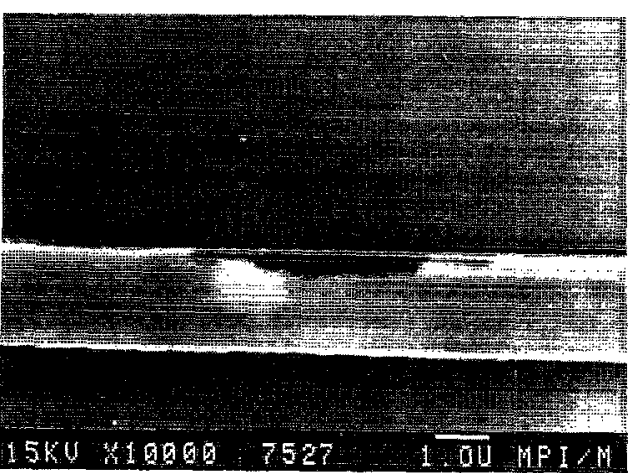

(d)

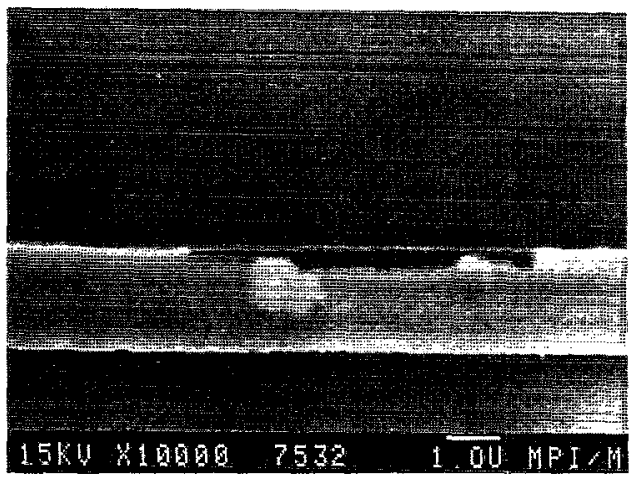

(e)
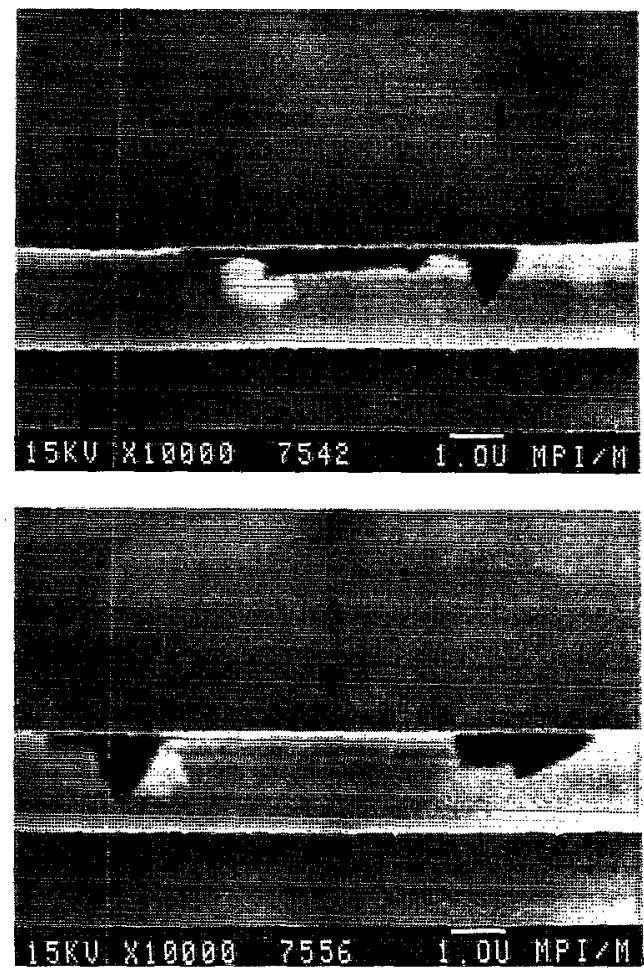

(g)
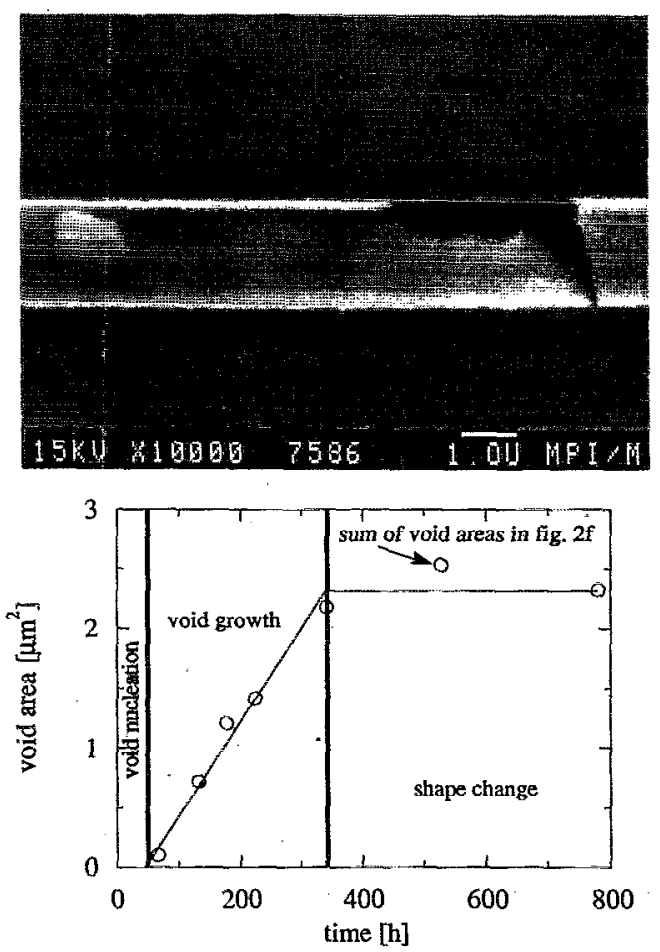

FIG. 2. Sequence of SEM micrographs similar to Fig. 1, testing time: (a) $65.8 \mathrm{~h}$, (b) $131.8 \mathrm{~h}$, (c) $177.2 \mathrm{~h}$, (d) $224.3 \mathrm{~h}$, (e) $341.9 \mathrm{~h}$, (f) $528.6 \mathrm{~h}$, and (g) 780.6 h. (h) Void area as a function of time.

configuration of a fatal void [Fig. 4(b)] was never observed in our studies. Certainly in an earlier stage of void growth (see Fig. 3) this shape can occur, but does not seem to be critical; instead the void has to change its shape in order to become fatal. The electron wind is expected to interact with the void shape in a way suggested qualitatively in Fig. 4: The driving force for electromigration on a void surface depends, to a first approximation, on the angle of this surface relative to the electron flow. Therefore a driving force does not appear on a surface perpendicular to the electron flow. Following this reasoning a void with a shape shown in Fig. 4(a) becomes "critical," because the electromigration-induced 


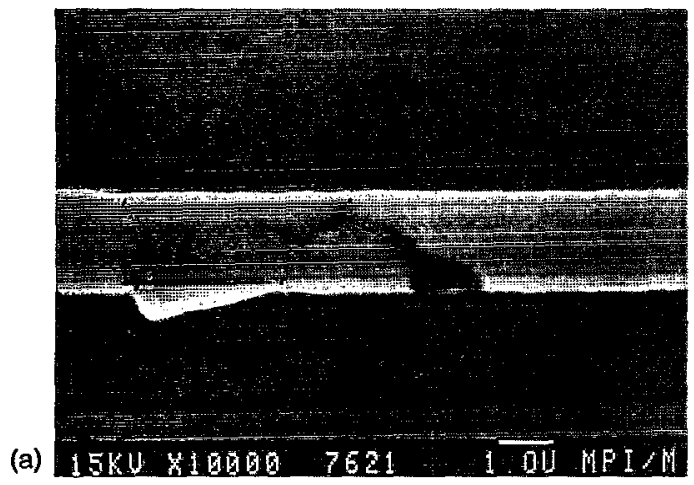

(a)
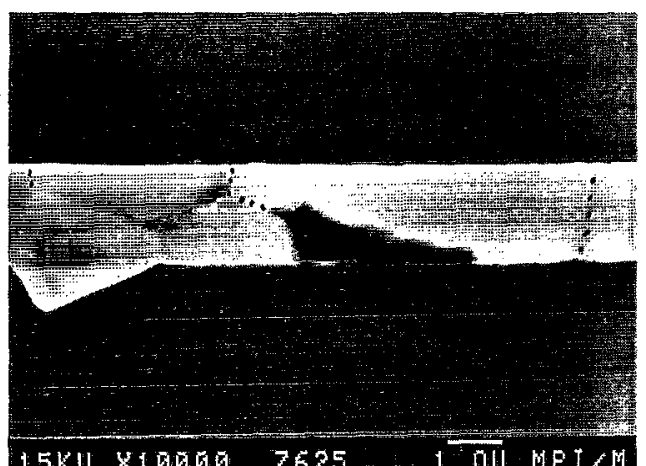

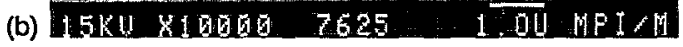

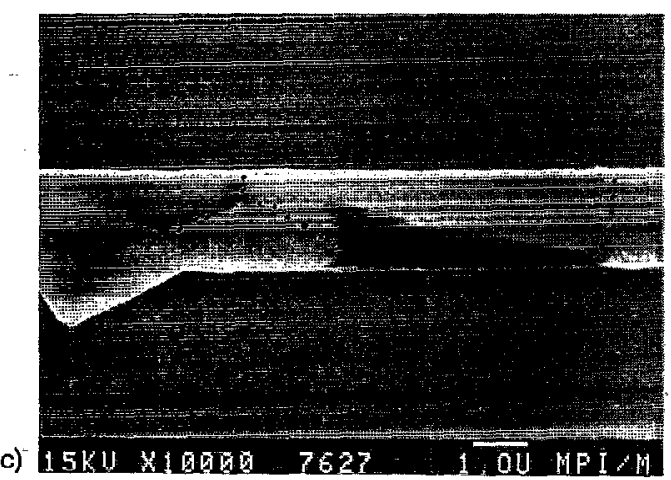

mass flux from to 2 to 1 is larger than from 3 to 2 . Consequently, mass is removed at 2 , the void grows across the line, and causes failure. Contrary to this, a void with a shape as in Fig. 4(b) should be uncritical, because electromigrationinduced mass flux from 3 to 2 is larger than from 2 to 1 . Thus

a) critical

b) uncritical
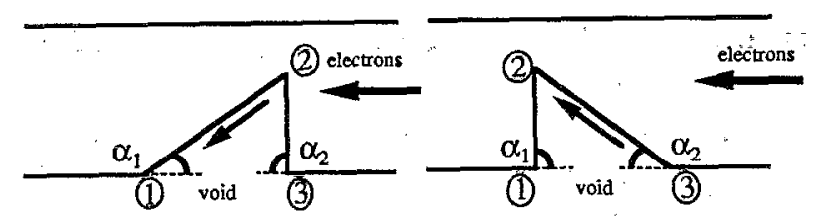

FIG. 4. Schematic illüstration of the interaction between the void shape and the electron wind: (a) Since $\alpha_{2}$ equals $90^{\circ}$, no electromigration induced mass flux from 3 to 2 but from 2 to 1 as a function of $\alpha_{1}$. Consequently mass is removed at 2 and the void tends to become fatal ("critical" void shape). (b) No electromigration-induced mass fiux on the void surface from 2 to 1 $\left(\alpha_{1}=90^{\circ}\right)$, but from 3 to 2 , as a result mass is removed at 3 and the void grows along the line ("uncritical" void shape).
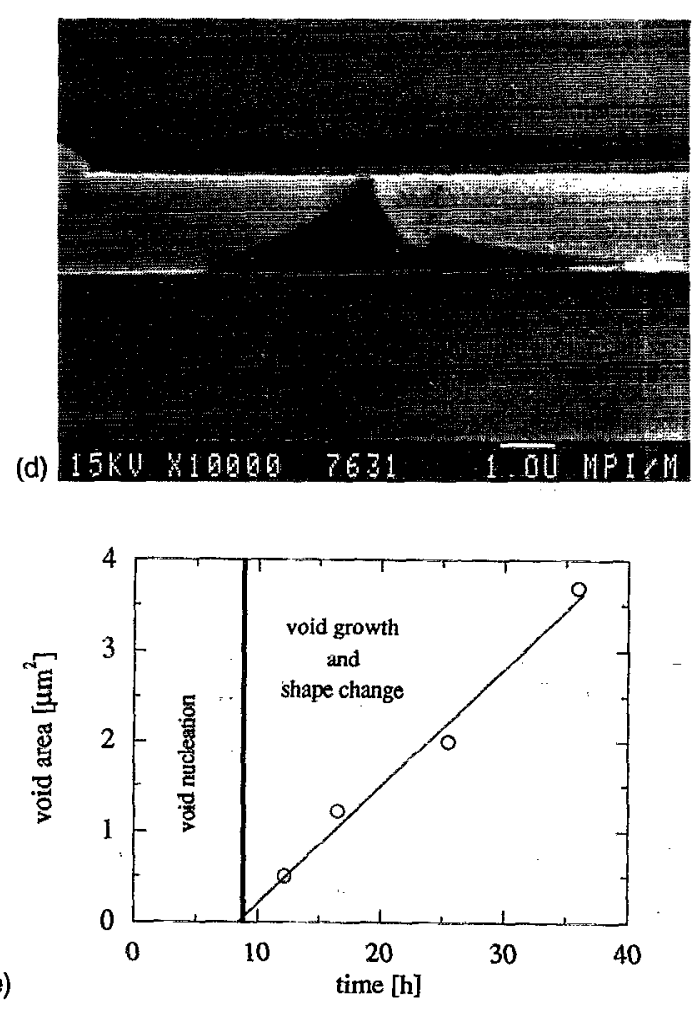

FIG. 3. SEM micrographs showing a time sequence on a conductor line of the "reflowed" film (grain boundaries are artificially highlighted): (a) 12.2 $\mathrm{h}$, (b) $16.5 \mathrm{~h}$, (c) $25.5 \mathrm{~h}$, and (d) $42.2 \mathrm{~h}$. (e) Void atea as a function of time.

the void grows along the line, like the void shown in Figs. 3(a) -3(c), until it changes the shape to a critical configuration as shown in Fig. 3(d).

This simple model is also consistent with Figs. 1 and 2 (and many other observations). Figure 1 shows a void with a critical shape, which produced the failure without any significant shape changes. Figure 2 illustrates a void with an uncritical shape until one part of the void broke away and moved along the line. This second void finally stopped moving and grew with a critical shape at the expense of the other fragment, producing the open circuit. The growth of the void to a certain size was not sufficient for failure of the line, a shape change was also necessary.

In summary, the following sequence of void behavior was observed:

(1) Void nucleation occurs at the line edge, probably where it is intersected by a grain boundary. Nucleation takes up only a small part of the lifetime.

(2) Void growth appears to be linear with time, as would be expected from electromigration kinetics at a constant cur- 

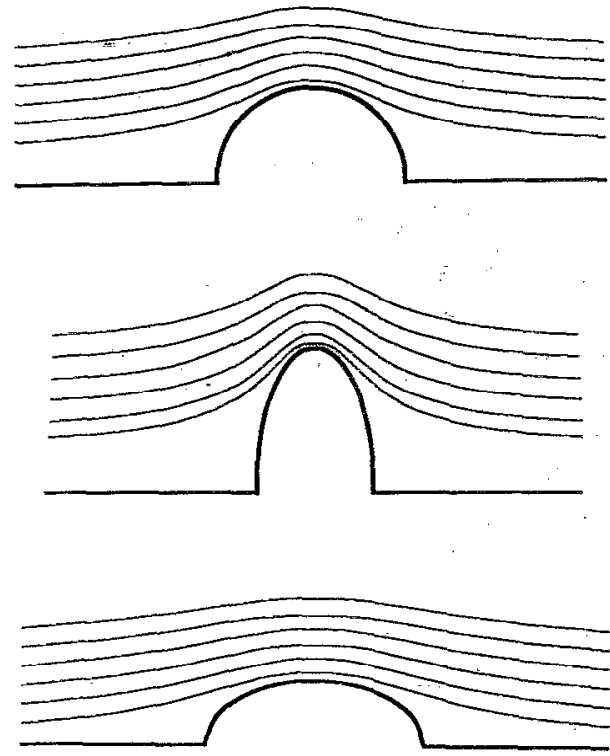

FIG. 5. Schematic of initial void shapes with resulting flowlines: (a) semicircular void, (b) transverse semi-elliptical void with $a<b$, and (c) longitudinal semi-elliptical void with $a>b$.

rent density. In order to continue growing, a void has to have a critical shape.

(3) Void motion is not always observed, but in several cases a void moved over some microns, opposite to the electron wind, until it became fatal.

(4) Shape change of a void is often the final step to produce the failure. It may take up a substantial fraction of the life time of a line.

At least two fundamental problems pose themselves in the light of these observations. First, why do the voids depart from an energetically favorable circular shape in the first place? And second, what is the driving force behind the characteristic asymmetry in critical voids? The first effect could be due to faceting because of anisotropic surface energy, as has been suggested before, e.g., by Refs. 17 and 26. The second, however, must be related to the electron wind. The following theoretical treatment of the competition between void motion and shape change suggests that both mechanisms may be the result of electron wind effects with the anisotropy of surface energy playing a minor role.

\section{MODELING VOID MOTION AND SHAPE CHANGE DUE TO THE ELECTRON WIND}

We wish to describe the motion and shape evolution of a void with a simple initial shape when subjected to a high current density. Based on experimental observations, we consider an isolated "two-dimensional" void which extends through the thickness of the line. As initial geometries we choose a circle [i.e., the equilibrium shape for a twodimensional (2D) void in a medium with isotropic surface energy] and an ellipse. These are possible model configurations for void nuclei, both in the interior and at the edge of a line as plotted in Fig. 5 .

We assume that the decisive mechanism is diffusion on the void surface and disregard the effects of grain boundaries

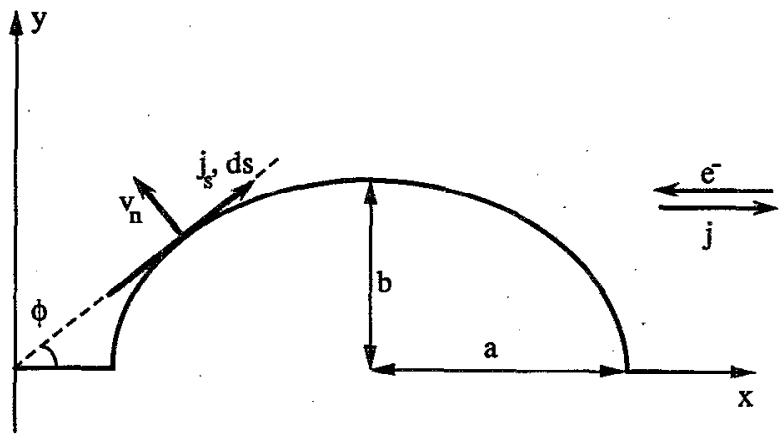

FIG. 6. Schematic illustration of the model geometry, where $j_{s}$ is the current component on the void surface, $d s$ the infinitesimal arc length along the surface, and $v_{n}$ the normal velocity of the surface.

and interfaces. This simplification is probably admissible for large-grain bamboo lines with natural or applied passivation. With reference to Fig. 6, the electron wind associated with a current density $j$ will cause surface atoms to diffuse from right to left. Depending on the balance of arriving and departing atoms at each point of the surface, this will cause the void to move (relative to a fixed coordinate system in the line) and/or to change its shape.

The subsequent analysis will address the following problems:

(i) Duie to the presence of the void, the initially homogeneous distribution of current density is perturbed. For the simple void shapes assumed, the components of the current density in the void surface can still be derived analytically by analogy to the hydrodynamic flow of an inviscid fluid (see Sec. IV A).

(ii) With the knowledge of the electron wind component at each point of the void surface, the surface fluxes can be derived. From the divergence of the surface flux at each point, the motion of the void surface with respect to the fixed coordinate system in the line is then calculated (see Sec. IV B). If the velocity of this motion is not identical at each location on the surface, a shape change of the void will result (see Sec. IV C).

What is neglected in this analysis is the fact that the inhomogeneous current distribution will cause temperature gradients with local changes in resistivity and diffusivity. These effects, which are believed to be potentially important for the failure mechanism, are beyond the scope of the present treatment.

\section{A. Distribution of current density in the presence of a void: The analogy with hydrodynamics}

Consider a 2D void in a conductor line and assume, for simplicity, that the void dimensions arc small compared to the linewidth (no edge effects). Since the electron flow lines cannot enter the void, an inhomogeneous current distribution is set up in the vicinity of the void. The equations of electrostatics, from which the flow lines can be calculated under the appropriate boundary conditions, are

$$
\nabla \cdot \mathbf{E}=0 \quad \text { and } \nabla \times \mathbf{E}=0,
$$


where $\mathbf{E}$ is the electric field vector. These expressions are mathematically identical to those for steady flow of an incompressible, non-viscous, circulation-free liquid: ${ }^{27}$

$$
\nabla \cdot \mathbf{v}=0 \text { and } \nabla \times \mathbf{v}=0 \text {, }
$$

where $\mathbf{v}$ is the velocity field vector, which corresponds directly to $\mathbf{E}$ in the electric analog, or through Ohm's law to $j$. In addition the boundary conditions imposed on the flow-lines by a void in an electrically conductive medium are analogous to those of fixed objects immersed in a liquid stream: in both cases the field components normal to the tangent of the void/object must vanish. This analogy enables us to draw on existing solutions for hydrodynamic flow past immersed objects whose shape corresponds to the shape of electromigration voids.

First, we consider a circular void as in Fig. 5(a). We seek the components $j_{s}$ of the current density along the perimeter of the void when the applied current density far away from the void is $j$. The analogous case is the streaming motion past a circular cylinder, from which we get (Ref. $28, \mathrm{p}$. 154)

$$
j_{s}=2 j \cos \phi,
$$

where $\phi$ is the angle between the line direction and the tangent to the pore surface. Thus the current density acting on the perimeter is simply the component of $j$ resolved in the direction of the tangent, but with an "amplification factor" of $A=2$. The same procedure can be applied to elliptic voids with the axis $a$ along the $x$ direction and $b$ along the $y$ direction. It is shown in the Appendix $\Lambda$ that $j_{s}$ is given by

$$
j_{s}=j \frac{(a+b) y}{\sqrt{y^{2}\left(a^{2}-b^{2}\right)+b^{4}}},
$$

where $y$ defines the point on the elliptical contour. This again corresponds to the component of $j$ resolved in the direction of the tangent to the void contour, amplified by a factor

$$
A=\frac{a+b}{a} \text {. }
$$

Note that the correct limit $A=2$ is obtained for a circle ( $a$ $=b)$. In the limit of a sharp crack $(a \rightarrow 0)$, a singularity develops in the current density distribution at the crack tip.

The void geometries discussed above are depicted, with their flow lines, in Fig. 5. The current density components in the void surfaces will be used to calculate the surface fluxes in the next section.

\section{B. Equation of motion for the void surface}

Following Black, ${ }^{1}$ the surface current of mass per unit film thickness, due to a current density $j_{s}$ parallel to that surfacc, is given by

$$
I_{s}^{e}=-\frac{\delta D_{s}}{\Omega k T} e Z^{*} \rho j_{s},
$$

where $e Z^{*}$ is the absolute value of the effective charge, $\rho$ the resitivity, $D_{s}$ the surface diffusivity, $\delta$ the thickness of the surface phase, $\Omega$ the atomic volume, $k$ Boltzmann's constant, and $T$ the absolute temperature. Because of surface tension, any gradients in surface curvature will promote mass fluxes to reduce these gradients. It is shown in Appendix B that the associated mass current (per unit film thickness) is

$$
I_{s}^{\kappa}=\frac{\delta D_{s} \gamma}{k T} \frac{\partial \kappa}{\partial s},
$$

where $\gamma$ is the surface tension, $\kappa$ the curvature, and $s$ the arc length along the surface. In the presence of these mass currents, the normal velocity $v_{n}$ at each point of the surface follows from a volume balance:

$$
v_{n} d t d s=\left[\left(I_{s}+\frac{\partial I_{s}}{\partial s} d s\right)-I_{s}\right] d t \Omega,
$$

where $I_{s}=I_{s}^{e}+I_{s}^{\kappa}$ and $d t$ is an infinitesimal time interval. The resulting equation of motion for the void surface is

$$
v_{n}=\Omega \frac{\partial I_{s}}{\partial s}=\Omega \frac{\partial}{\partial s}\left(-a_{j} \frac{j_{s}}{j}+a_{\kappa} \frac{\partial \kappa}{\partial s}\right) .
$$

The new constant $a_{j}$ relates to current density

$$
a_{j}=\frac{\delta D_{s}}{\Omega k T} e Z^{*} \rho j
$$

while $a_{\kappa}$ contains the surface tension

$$
a_{\kappa}=\frac{\delta D_{s} \gamma}{k T}
$$

\section{Shape stability of different void geometries}

First, consider the behavior of a circular void with radius a. By combining Eqs. (3) and (9) and noting that $\partial \kappa / \partial s=0$, we get the classical result ${ }^{19}$

$$
v_{n}=-\frac{2 \Omega a_{j}}{a} \cos \theta
$$

As shown in Fig. 7(a) the shape of the circle remains fixed after a first infinitesimal time interval. Thus a circular void will only move against the electron flow, without any shape changes.

Next, we treat the case of an elliptic void with the $a$ axis parallel to the line. To ensure analytical tractability, we ignore diffusional currents due to curvature gradients, i.e., we require $a_{j} \geqslant a_{\kappa} / a^{2}$. In the same way as above, but after considerable algebra, the following result is obtained:

$$
v_{n}=-\frac{\Omega a_{j}}{a^{4}}(a+b) b^{2} \cdot(x / a) /\left[1-\left(1-\frac{b^{2}}{a^{2}}\right) \frac{x^{2}}{a^{2}}\right]^{2} .
$$

The resulting shape evolution in the first infinitesimal time interval is depicted in Figs. 7(b) and 7(c) for transverse ( $a$ $<b)$ and longitudinal $(a>b)$ ellipses. It is striking that a transverse ellipse suffers a shape distorlion which tends to produce both a flatter face at the cathode end and a slight lateral growth of the void. This result is in qualitative agreement with our experimental observations. By contrast, the longitudinal ellipse tends to elongate towards the cathode and shows no lateral growth. It stands to reason that a void with an initial shape close to the first case tends to become fatal under the action of the electron wind, whereas a void 
(a)

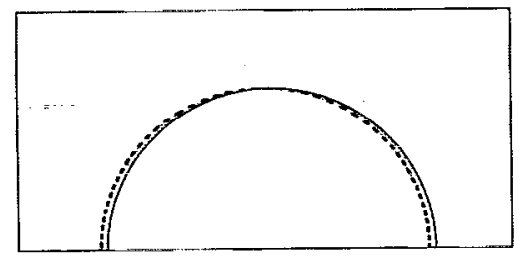

(b)

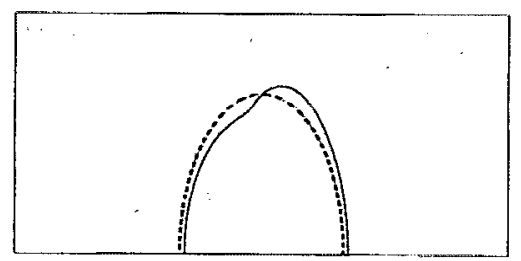

(c)

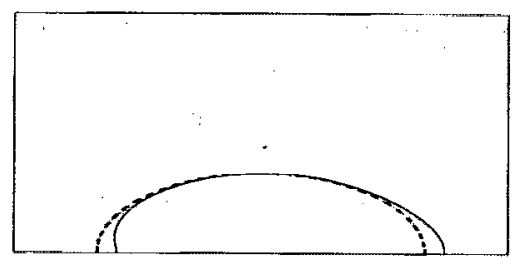

FIG. 7. Calculated void shape changes for three different initial void shapes (dotted line: initial shape, electron flow from the right to the left): (a) circular void: the void moves without shape change, (b) elliptical void with $a<b$ : the void tends to form a flat facet at the cathode end and shows a slight lateral growth, (c) elliptical void with $a>b$ : the void tends to elongate and shows no lateral growth.

with a shape close to latter case would grow along rather than across the line. Figures 2 and 3 may be such cases in point.

This result might also explain other apparently contradictory observations: sometimes voids are shown to grow along the lines, ${ }^{8,12}$ whereas often void growth occurs perpendicular to the electron flow. ${ }^{7,14}$ Both observations could be attributed to the influence of the initial void shape described. However, these results should still be considered as preliminary. In our analytical treatment only the initial stage of the shape evolution can be covered; the resulting deviations from simple elliptic shapes increase the complexity of the electron flow field, which is in addition influenced by edge effects. Also, we have not considered the effects of diffusion due to curvature gradients; these may tend to stabilize the void shape to an extent that is presently unknown.

\section{CONCLUDING REMARKS}

Our observations point to the importance of void shape changes as an element in the development of fatal damage in bamboo interconnect lines under electromigration conditions. These processes can take up more than half of the lifetime of an unpassivated interconnect. A critical shape was identified: the void has to develop a more or less flat facet perpendicular to the line at the cathode end. A model was presented which describes how a void with a simple initial shape changes its shape and moves under the action of the electron wind. These calculations show that it is not necessary to assume anisotropic surface energies in order to simulate initial shape changes in the right direction. Instead, such shape changes may be due to the dynamics of the electron wind effect, in a similar way to the instability described recently by Suo. ${ }^{29}$ This conclusion seems to be supported by our recent in situ SEM observations ${ }^{30}$ which show that the facetting of voids disappears when the electric current is turned off. Thus, the anisotropy of the surface energy may possible play only a minor role in the shape change.

Void shape change as a failure mechanism may also explain why the lifetime of a pre-damaged line can be much shorter than that of an undamaged line, as found by Lytle and Oates. ${ }^{31}$ It is conceivable that pre-existing voids formed, e.g., by stress-induced vacancy migration, may change shape under electromigration conditions and produce the failure without any further growth. In this case neither void nucleation under electromigration conditions nor long-range mass transport is necessary.

In order to arrive at a more complete understanding, other driving forces for void shape changes than the electrical current have to be considered in more detail, e.g., effects of anisotropic surface energies and mechanical stresses. Detailed numerical simulations including temperature distribution, stress and edge effects, combined with in situ observations of the voiding process, are currently in progress. ${ }^{30}$

\section{ACKNOWLEDGMENTS}

This article is the result of an ongoing collaboration between University of Stuttgart and Stanford University. EA and WDN are grateful for financial support in the form of the Max-Planck Prize, JES acknowledges support by the MaxPlanck Society during his stay as a Visiting Scientist in Stuttgart. OK would like to thank AMD for supporting a research visit at Sunnyvale.

\section{APPENDIX A: CURRENT DENSITY ALONG THE CONTOUR OF AN ELLIPTIC VOID}

From the hydrodynamics of flow past an elliptic cylinder, with its major axis oriented at angle $\alpha$ to the stream (Ref. 28, p. 165), we get for $j_{s}$ :

$$
j_{s}^{2}=j^{2} \frac{a+b}{a-b} \cdot \frac{\sinh ^{2}\left(\xi-\xi_{0}\right)+\sin ^{2}(\eta-\alpha)}{\sinh ^{2} \xi+\sin ^{2} \eta},
$$

where, as in Ref. 28 , the cylindric coordinates $\xi=\xi_{0}$ and $\cos \eta=x / a$ define the elliptic contour with $a$ and $b$ as major and minor axis and $\xi_{0}=(1 / 2)[\log (a+b)-\log (a-b)]$. This expression can be transformed into

$$
j_{s}=j(a+b) \sin (\eta-\alpha) / a \sqrt{1-\left(\frac{x}{a}\right)^{2}\left(1-\frac{b^{2}}{a^{2}}\right)},
$$

which, for $\alpha=0$, reduces to Eq. (4) in the text. It is instructive to show that Eq. (4) gives a surface current density which is proportional to the component of $j$, resolved in the direction of the tangent to the surface contour. Claiming that

$$
j_{s}=j \cos \phi,
$$

where $\phi$ is the angle between the tangent and the line direction, we obtain after some algebra: 


$$
j_{s}=j \frac{a y}{\sqrt{y^{2}\left(a^{2}-b^{2}\right)+b^{4}}} .
$$

This expression differs from Eq. (4) in the text only by a constant amplification factor of $A=(a+b) / a$.

\section{APPENDIX B: FLUXES DUE TO CURVATURE GRADIENTS}

The curvature at any point of a surface can be expressed as

$$
\kappa=-\frac{y^{\prime \prime}}{\left[1+\left(y^{\prime}\right)^{2}\right]^{3 / 2}}=-\frac{d \alpha}{d s},
$$

where $\alpha$ is the angle between the surface tangent and the $x$-axis, $y^{\prime}$ and $y^{\prime \prime}$ are derivatives of the surface shape $y(x)$, and the curvature is defined as positive for a concave void surface. is

The atomic chemical potential, relative to a flat surface,

$$
\Delta \mu=-\Omega \gamma \kappa \text {. }
$$

Because the diffusional flux is generally given by

$$
J_{s}^{\kappa}=-\frac{D_{s}}{\Omega k T} \frac{\partial \mu}{\partial s},
$$

we obtain for the mass current (per unit film thickness)

$$
I_{s}^{\kappa}=\frac{\delta D_{s} \gamma}{k T} \frac{\partial \kappa}{\partial s} \text {. }
$$

${ }^{1}$ J. R. Black, IEEE Trans. Electron Devices ED-16, 338 (1969).

${ }^{2}$ R. E. Hummel, International Materials Reviews (in press).

${ }^{3}$ M. Shatzke and J. R. Lloyd, J. Appl. Phys. 59, 3890 (1986).

${ }^{4}$ E. Arzt and W. D. Nix, J. Mater. Res. 6, 4113 (1991).
${ }^{5}$ M. J. Attardo, R. Rutledge, and R. C. Jack, J. Appl. Phys. 42, 4343 (1971). ${ }^{\circ} \mathrm{J}$. R. Lloyd, J. Appl. Phys. 69, 7601 (1991).

${ }^{7}$ R. W. Thomas and D. W. Calabrese, Proceedings of the 21 st Annual IEEE International Reliability Physics Symposium, 1983, pp. 1-9.

${ }^{8}$ S. Shingubara and Y. Nakasaki, Appl. Phys. Lett. 58, 42 (1991).

${ }^{9}$ E. Castano, J. Maiz, P. Flinn, and M. Madden, Appl. Phys. Lett. 59, 129 (1991).

${ }^{10}$ P. R. Besser, M. C. Madden, and P. A. Flinn, J. Appl. Phys. 72, 3792 (1992).

${ }^{11}$ M. C. Madden, E. V. Abratowski; T. Marieb, and P. A. Flinn, MRS Symp. Proc. 265, 33 (1992).

${ }^{12}$ I. Vávra and P. Lobotka, Phys. Status Solidi A 65, K107 (1981).

${ }^{13}$ R. W. Vook and C. Y. Chang, Appl. Surf. Sci. 60/61, 71 (1992).

${ }^{14}$ O. Kraft, S. Bader, J. E. Sanchez, Jr., and E. Arzt, MRS Symp. Proc. 309, 199 (1993).

${ }^{15}$ J. E. Sanchez, Jr. and E. Arzt, MRS Symp. Proc. 265, 131 (1992).

${ }^{16}$ J. E. Sanchez Jr., O. Kraft, and E. Arzt, Appl. Phys. Lett. 61, 3121 (1992).

${ }^{17}$ J. H. Rose, Appl. Phys. Lett. 61, 2170 (1992).

${ }^{18}$ J. H. Rose and T. Spooner, MRS Symp. Proc. 309, 409 (1993).

${ }^{19}$ P. S. Ho, J. Appl. Phys. 41, 64 (1970)

${ }^{20}$ W. D. Nix and E. Arzt, Met. Trans. A 23A, 2007. (1992)

${ }^{21}$ P. Børgesen, M. A. Korhonen, T. D. Sullivan, D. D. Brown, and C.-Y. Li, MRS Symp. Proc. 239, 683 (1991).

${ }^{22}$ C. V. Thompson and J. Cho, IEEE Electron Device Lett. 7, 667 (1986).

${ }^{23}$ J. E. Sanchez, Jr., L. T. McKnelly, and J. W. Morris, Jr., Electron. Mater. $19,1213(1990)_{\varepsilon}$

${ }^{24} \mathrm{~S}$. Chen and E. Ong, Proceedings of Society of Photoptical Integrated Engineering 1989 Symposium on Microelectronic Integrated Processing: Conference on Laser/Optical Processing of Electronic Materials, Santa Clara, CA, Oct. 10-11, 1989.

${ }^{25}$ K. Nikawa, J. Vac. Sci. Technol. B 9 (5), 2566 (1991).

${ }^{26}$ M. Hasunuma, H. Kaneko, A. Sawabe, T. Kawanoue, Y. Kohanawa, S. Komatsu, and M. Miyauchi, Proceedings of the Annual Meeting IEDM IEEE, Washington, DC, 1989 , pp. 677-680.

${ }^{27}$ R. P. Feynman, R. B. Leighton, and M. Sands, Lecture on Physics, Vol. II (Addison-Wesley, Reading, MA, 1966), 4th printing, p. 12-8.

${ }^{28}$ L. M. Milne-Thomson, Theoretical Hydrodynamics (Macmillan, New York, 1963), 4th printing.

${ }^{29} \mathrm{Z}$. Suo (personal communications).

${ }^{30}$ E. Arzt, O. Kraft, and U. Möckl, Materials Research Society Spring Meeting, San Francisco, April 1994.

${ }^{31}$ S. A. Lytle and A. S. Oates, J. Appl. Phys. 71, 174 (1992). 